\title{
Self-Relatedness and Interpersonal Problems in a Large Psychiatric Outpatient Sample
}

\author{
Espen Bjerke', Ole A. Solbakken², Svein Friis ${ }^{3}$, Jon T. Monsen² \\ ${ }^{1}$ Department of Psychiatry, $\emptyset$ stfold Hospital, Sarpsborg, Norway \\ ${ }^{2}$ Department of Psychology, University of Oslo, Oslo, Norway \\ ${ }^{3}$ Faculty of Medicine, University of Oslo, Oslo, Norway \\ Email: espbje@so-hf.no
}

Received 10 May 2016; accepted 13 June 2016; published 16 June 2016

Copyright (C) 2016 by authors and Scientific Research Publishing Inc.

This work is licensed under the Creative Commons Attribution International License (CC BY). http://creativecommons.org/licenses/by/4.0/

(c) (i) Open Access

\begin{abstract}
Interpersonal- and self-relatedness problems are strongly correlated on a global level. However, few studies have examined associations between distinct forms of interpersonal problems and specific types of problems in self-relatedness. We hypothesized that patients with domineering interpersonal problems would exhibit a more positive self-image than patients with submissive interpersonal features. We also wanted to examine if the self-hostile and self-accepting aspects of patients' self-relatedness were differentially associated with their interpersonal problems. A large clinical outpatient sample $(\mathrm{N}=958)$ was divided into eight subgroups (Octant Groups) of patients with different predominant interpersonal problems, as measured with the 64 item version of the Inventory of Interpersonal Problems-IIP-64. Self-relatedness was measured with the Introject surface of the Structural Analysis of Social Behavior-SASB Introject. ANCOVA analyses showed that patients with more positive and self-accepting self-images displayed domineering, but also warmer forms of interpersonal problems than peers with more negative self-images. Dividing the self-relatedness variable into Self-Hostile and Self-Accepting components yielded a new finding: patients within different interpersonal Octant Groups differed significantly with regard to SelfAcceptance, but not with respect to Self-Hostility. The former component may be reflective of a more stable personality feature than Self-Hostility, thereby rendering it less susceptible to change.
\end{abstract}

\section{Keywords}

Interpersonal Problems, Self-Image, Self-Relatedness, Personality, Psychiatric Patients 


\section{Introduction}

Interpersonal theory holds that individuals often treat themselves as others have treated them (Horowitz, 2004). Persistent criticism from significant others is likely to foster self-criticism. Internalized self-criticism may, however, manifest itself in different ways in the interpersonal domain, as repetitive patterns of criticizing others or inviting criticism from others. Maladaptive interpersonal styles may develop into interpersonal problems and, eventually, personality disorders. In addition, internalized self-criticism may lead to symptoms, such as depression, shame, and guilt (Horowitz, 2004).

In related literature, there is a general agreement that interpersonal and self-relatedness problems are strongly correlated on a global level and that both are robustly related to measures of psychopathology (Horowitz et al., 2006). However, very few studies have examined specific associations between interpersonal problems/styles and problems of relating to the self (Monsen, von der Lippe, Havik, Halvorsen, \& Eilertsen, 2007; Pincus, Gurtman, \& Ruiz, 1998; Ruiz et al., 2004). The present study addresses this gap in the literature by examining specific associations between self-relatedness and interpersonal functioning in a large clinical sample, and by discussing potential implications for diagnosis and treatment planning. The 64-item Inventory of Interpersonal Problems (IIP-64; Horowitz, Alden, Wiggins, \& Pincus, 2000) is used to measure interpersonal problems, and the introject surface of Structural Analysis of Social Behavior (SASB Introject) is used to measure various aspects of self-relatedness (Benjamin, 1974).

\subsection{The Interpersonal Circumplex}

According to interpersonal theory, human behaviors and traits are shaped by two fundamental desires, to "get along” and "get ahead” (Pincus \& Gurtman, 2006). Harry Stack Sullivan, considered the father of interpersonal theory, claimed that social relations are at the heart of psychopathology (Sullivan, 1953). In a series of articles by Leary and colleagues, it was demonstrated that interpersonal mechanisms can be arranged in a circular manner (Leary, 1957). These works were followed by a number of studies that showed that interpersonal functioning may be conceptualized within the interpersonal space. The term "Interpersonal Circumplex" (IPC) was coined by Wiggins $(1979,1996)$, who merged the work of Leary's group with the psychometric model proposed by Guttman (Gurtman, 2009; Guttman, 1954). The original 127-item version of the Inventory of Interpersonal Problems (IIP) was developed by Horowitz, Rosenberg, Baer, Ureno, and Villasenor (1988). In an effort to reduce respondent burden, Alden, Wiggins, and Pincus (1990) developed a shorter version of the IIP, the Inventory of Interpersonal Problems-64 (IIP-64), with only 64 items.

One of the most exciting features of the interpersonal circumplex is its capacity to depict interpersonal phenomena with reference to the geometric properties of a circle, with two dimensions defining Cartesian space (i.e., Communion or Affiliation as the horizontal dimension and Agency or Dominance as the vertical dimension). An interpersonal profile can be reduced to a summary point in the two-dimensional interpersonal space, and a vector from the origin to this point reflects the overall trend of the profile.

Figure 1 depicts the following core features of the IPC: the two main dimensions, Communion (COM) and Agency (AGE); the angle in degrees (see the Method section), and the name of the eight subscales. Capital letters from A to P refer to the 16 interpersonal mechanisms that were originally proposed by Leary's group and continue to be employed. This allows for rapid communication across specific IPC measures.

\subsection{Structural Analysis of Social Behavior (SASB)}

The SASB model, not unlike the IIP, is based on interpersonal/object relational tradition (Mahler, Pine, \& Bergman, 1975; Sullivan, 1953) and the tradition of interpersonal circumplex models (Benjamin, 1996; Leary, 1957). The original SASB model is more complex than other two-dimensional circumplex models in that it includes three surfaces: 1) interpersonal focus on others, or my actions toward others (transitive, “parent-like,” action); 2) interpersonal focus on the self, or my reactions to others’ actions against me (intransitive, “infant-like,” action); and 3) introjected actions directed toward myself. This third surface, the "introject," reflects a hypothesized personality structure of a relatively stable repertoire of actions and attitudes toward oneself and is assumed to be formed by interpersonal experiences that are internalized early in life (Benjamin, 1996). Each surface is defined by dimensions of Affiliation and Interdependence. The SASB Introject surface, which forms the basis of the self-relatedness measures employed in this study, includes eight clusters that are positioned in a circular arrangement, representing combinations of the Affiliation and Interdependence dimensions (cf. Figure 2). 


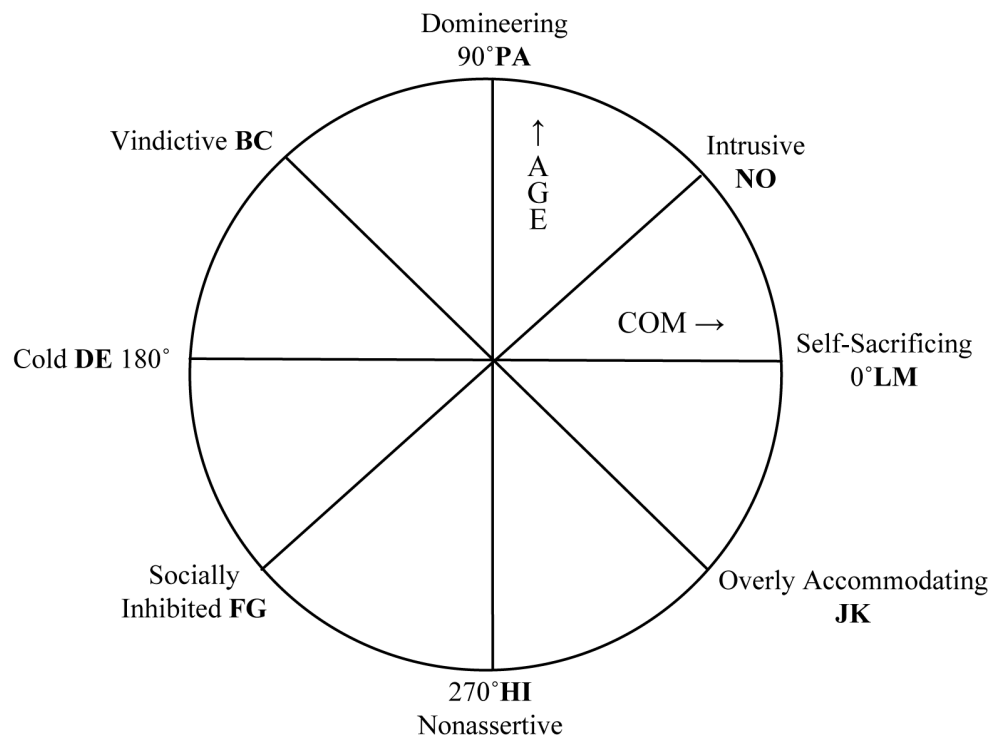

Figure 1. The Interpersonal circumplex. Eight subscales of the IIP-64. Two dimensions: Communion and Agency.

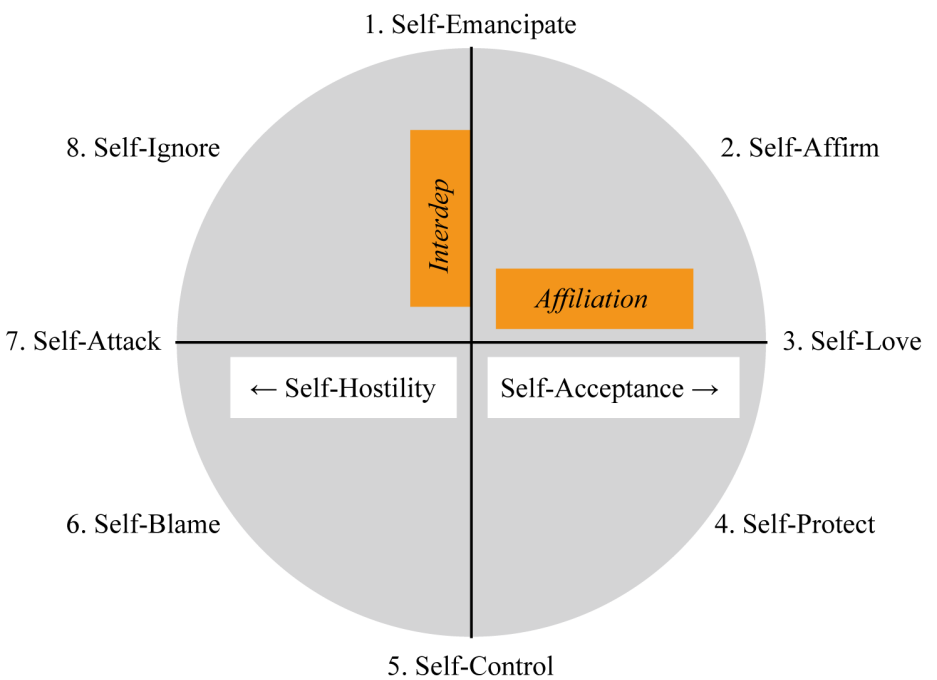

Figure 2. Eight Clusters denoting different forms of self-relatedness. Two dimensions: Affiliation and Interdependence. Two aspects of the Affiliation dimension: Self-Acceptance and Self-Hostility.

Several studies have found a strong relationship between the (horizontal) Affiliation dimension of the SASB Introject and psychopathology. Higher levels of Affiliation are associated with lower levels of emotional distress and vice versa (Henry, 1996; Monsen et al., 2007; Pincus et al., 1998). The relationship between the Interdependence dimension and psychopathology has been examined less frequently (Granberg \& Armelius, 2003; Monsen et al., 2007). In a previous study on a student sample, Pincus et al. (1998) found that higher scores of Affiliation correlate with a tendency toward dominance in the interpersonal domain, indicating that positive forms of self-relatedness do not necessarily run parallel to positive forms of relating to others. This result may also indicate the inverse association, that is, that negative forms of self-relatedness are likely to correlate with interpersonal submissiveness. This would imply a kind of "non-parallelism" between the IIP and SASB models, which we wish to scrutinize in the present study.

Components of Affiliation: Self-Acceptance and Self-Hostility. Clinical studies on the Affiliation dimension 
of the SASB Introject have mainly assessed the overall Affiliation vector. Some studies, however, have recommended splitting this measure into disaffiliative and affiliative, or Self-Hostile and Self-Accepting, components. Hilliard, Henry, and Strupp (2000) stated that "collapsing across disaffiliative and affiliative codes in the SASB codings largely masks the variability in disaffiliative codes, given that they compose a relatively small percentage of the total number of codes. Previous research (Henry et al., 1986, 1990) has shown, however, that even small amounts of disaffiliative process have a significant deleterious effect on outcome” (p. 128). Other studies have shown that measures of Self-Acceptance and Self-Hostility present unique characteristics, such as different patterns of change during therapy (Granberg \& Armelius, 2003). Halvorsen and Monsen (2006) found that changes in Self-Acceptance and Self-Hostility during psychotherapy are related but not mirror images of one another. Their findings showed that a decline in Self-Hostility is easier to achieve and occurs earlier in the treatment process, whereas changes that promote Self-Acceptance might reflect a more complex process that requires a longer period of time. Figure 2 presents the main dimensions of the SASB Introject circle and the two components of the Affiliation dimension.

\subsection{Research Hypotheses and Aims}

First, previous research has shown that subcomponents of the Affiliation dimension in the SASB Introject surface, Self-Hostility and Self-Acceptance, may not only be interpreted as two extreme points on the same dimension but also as representing separate features of self-relatedness. In the present study, we examine these indices separately to determine whether they are differentially associated with interpersonal problems.

Second, we assume that self-reports from psychiatric outpatients will display more negative forms of selfrelatedness, as reflected in the SASB Introject, than those of the general population. The most significant differences are expected along the Affiliation dimension, that is lower Self-Acceptance (Clusters 2 - 4) and higher levels of Self-Hostility (Clusters 6 - 8).

Third, we examine associations between the patients' Affiliation scores and salient features of their interpersonal problems (derived from a structural summary of IIP-profiles, see the Methods section). These features include the global level of interpersonal problems (IIP Global), the rigidity of interpersonal style (Intensity), and the location of problems within the IPC (Octant Group). We expect all of these interpersonal variables, in addition to general symptom distress levels (GSI see the Methods section), to contribute significantly to the variance in the self-relatedness variables (i.e. Affiliation and its subcomponents Self-Acceptance and Self-Hostility).

Fourth, in connection with the claim that positive forms of self-relatedness not necessarily run parallel to positive forms of relating to others), we examine whether patients with predominant interpersonal problems in the Intrusive, Domineering, and Vindictive Octant Groups have more positive forms of self-relatedness and higher Affiliation scores than their Socially Inhibited, Nonassertive and Overly Accommodating peers.

\section{Method}

\subsection{Participants-Outpatient Sample}

The sample consisted of consecutively admitted patients who were offered initial examination/treatment at an outpatient psychiatric clinic in Moss, Norway. The clinic covers a population of 60,000 persons. Exclusion criteria: Emergency cases, non-Norwegian-speaking patients, and patients who had previously been offered/given treatment at the clinic. During the study period close to 4000 persons were admitted to the unit. Previously registered patients (25\%), emergency cases (25\%), and non-Norwegian-speaking patients (10\%) were the largest groups among those not included in the study. Nine percent were excluded for other reasons, and this proportion primarily included patients who requested ADHD evaluations. Close to 1250 patients were sent a booklet by mail and asked to complete a number of self-report questionnaires, including the SASB Introject (36 items), IIP-64, and Symptom Check List 90 items-Revised version (SCL-90-R; Derogatis, 1983), before their first consultation at the clinic. A total of 988 patients submitted the forms, resulting in a response rate of $79 \%$ of those approached. The mean age of the patient sample was 34 years, with patients ranging from 16 to 77 years. Women comprised $64 \%$ of the sample.

Thirty patients were excluded from the study because they had returned incomplete SASB reports (Cluster scores were deemed incomplete when $\geq 50 \%$ of the items in a certain Cluster were missing). The mean age of the 30 patients who submitted incomplete reports was 35 years, and their global IIP and SCL-90-R/GSI scores 
were $1.54(0.44)$ and $1.72(0.75)$, respectively.

\subsection{Instruments}

\subsubsection{Structural Analysis of Social Behavior (SASB)}

The Norwegian version of the SASBIntroject Surface Long Form A was employed (Monsen et al., 1999). It includes 36 items that describe attitudes and feelings that one can have toward oneself. In the original SASB questionnaire, participants are asked to rate themselves in terms of their "best" and "worst" states. In this study, the instruction is changed to the following: "rate yourself as you usually are." This instruction was given in an effort to measure stable, trait-like aspects of self-relatedness. This instruction is the most commonly used with the Norwegian version of the SASB (Halvorsen \& Monsen, 2007; Svartberg, Seltzer, \& Stiles, 1996). SASB items are grouped into eight Clusters (Clusters 1, 3, 5, and 7 with five items; Clusters 2, 4, 6, and 8 with four items) that can be arranged in a circumplex within a two-dimensional space defined by the AffiliationandInterdependence dimensions. By convention, the Clusters are arranged clockwise, beginning with Cluster 1 at "twelve o'clock" (cf. Figure 2). Item statements, such as "I think of ways to hurt and destroy myself. I am my own worst enemy" (Cluster 7-Self-Attack), are rated on a scale from 0 (never, not at all) to 10 (always, perfectly). The Cluster scores presented are mean scores, or sum scores divided by the number of items in the respective Cluster ${ }^{1}$. In the present study, only the horizontal Affiliation dimension of the SASB Introject is examined.

The following equation is used to calculate overall Affiliation levels:

$$
\begin{aligned}
\text { Affiliation }= & (0 * \text { Cluster } 1+4.5 * \text { Cluster } 2+7.8 * \text { Cluster } 3+4.5 * \text { Cluster } 4) \\
& -(\text { minus })(0 * \text { Cluster } 5+4.5 * \text { Cluster } 6+7.8 * \text { Cluster } 7+4.5 * \text { Cluster } 8)
\end{aligned}
$$

(Pincus, Newes, Dickinson, \& Ruiz, 1998).

In addition, the positive (I) and negative (II) components of Affiliation, entitled Self-Acceptance and SelfHostility are examined separately. Affiliation is calculated as a difference score, and such scores may tend to compound error variance disproportionally, thereby reducing the reliability of a difference-score measure. Previous findings indicate that the Self-Acceptance and the Self-Hostility subcomponent of Affiliation may have unique characteristics.

An examination of Norwegian SASB Introject scores suggests fairly strong construct validity for the clinical samples but not for the normal reference sample. Presumably, this difference is of negligible consequence to our use of the instrument in evaluating self-relatedness in the present context (Monsen et al., 2007). Cronbach's alpha values for the eight Clusters employed in the present study ranged from 0.39 (Clusters 1 and 4) to 0.77 (Cluster 6), with a median of 0.72 . Only Clusters 1 and 4 had Cronbach's alpha values of $<0.70$.

\subsubsection{Inventory of Interpersonal Problems, 64-Item Version (IIP-64)}

The 64-item self-report Inventory of Interpersonal Problems (IIP-64) was used for the assessment of interpersonal problems. The IIP-64 includes 39 items that begin with the phrase: "It is hard for me to..." The remaining 25 items open with the following heading: "Things that you do too much.” Each item is rated on a 5-point Likert scale ranging from 0 (not at all) to 4 (extremely). Eight specific interpersonal styles or problems are presented and arranged in a circular fashion based on their angular position within the two-dimensional circumplex space (Horowitz et al., 2000). Octants and the corresponding scales include the following: Domineering/Controlling (PA), Vindictive/Self-Centered (BC), Cold/Distant (DE), Socially Inhibited (FG), Nonassertive (HI), Overly Accommodating (JK), Self-Sacrificing (LM), and Intrusive/Needy (NO). In the present study, the "short versions" of these categories are utilized (i.e., Domineering rather than Domineering/Controlling; Vindictive rather than Vindictive/Self-Centered, etc.). An examination of the Norwegian version of the IIP-64 applied in this study suggests acceptable reliability, strong internal structure, and discriminant and convergent validity with external criteria (Monsen, Hagtvet, Havik, \& Eilertsen, 2006). Cronbach's alpha values for the IIP subscales ranged from 0.64 to 0.84 , with a median of 0.77 (Bjerke, Solbakken, \& Monsen, 2014).

\footnotetext{
${ }^{1}$ Originally, Cluster scores were given as "percentage of maximal attainable" scores, which are equal to ten times the Cluster scores presented in the present study (Benjamin, 1974, 1996).

${ }^{2}$ Similar results can be attained by multiplying Clusters 2, 4, 6 and 8 by $0.71\left(\cos 45^{\circ}\right)$ rather than by 4.5 and then multiplying Clusters 3 and 7 by 1.00 rather than by 7.8. Following Pincus et al. (1998), we utilized Benjamin's original formula.
} 


\subsubsection{Structural Summary of IIP-64}

We employed the structural summary method proposed by Gurtman and Balakrishnan (1998) and thus represented individual IIP-64 profiles based on three parameters. The first parameter is the mean level (i.e., the sum of all item scores, divided by 64) of interpersonal distress (elevation) (hereafter, we use the term IIP Global for this parameter). The second parameter is the intensity of predominant interpersonal problems (amplitude). This parameter is based on vector and trigonometric calculations of the subscale scores (Gurtman \& Balakrishnan, 1998). Hereafter, we use the term Intensity for this parameter. The third parameter, also based on trigonometric calculations, yields the location of the individual's profile peak in interpersonal space, denoting predominant interpersonal problems (technically referred to as the Angle or Angular Displacement); stated in degrees $\left({ }^{\circ}\right)$ of displacement from the starting point. The degree of Angular Displacement is used as the basis for Octant Group assignment. By convention, an Angle of $0^{\circ}$ is placed in the Self-Sacrificing (LM) sector of the interpersonal circle, with increasing figures running counterclockwise (Figure 1).

In addition, the structural summary includes a Goodness of Fit (GOF) index (not addressed in this study), derived from a calculation of best fit scores, reflecting the degree of fit with an ideal, prototypic profile (Bjerke, Hansen, Solbakken, \& Monsen, 2011; Gurtman \& Balakrishnan, 1998; Gurtman \& Pincus 2003).

Using the structural summary method, one can thus determine, in addition to the IIP Global, each individual's substantive interpersonal problem (Octant Group), and the Intensity of his or her interpersonal problems (Wright, Pincus, Conroy, \& Hilsenroth, 2009).

Each participant was assigned to an Octant Group based on his/her self-reported pre-treatment interpersonal problems. The significance of this attribution to a specific Octant Group diminishes when the Intensity of problems is low. This is not to say that patients with low-Intensity issues do not have interpersonal problems. Rather, their problems may be of a more generalized nature. Patients may report very high levels of interpersonal problems in general, yielding high IIP Global scores. However, if their problems are evenly distributed across Octants of the interpersonal circle, this will be reflected as a low Intensity score in specific IIP-64 domains.

\subsubsection{Symptom Check List-90 Items-Revised (SCL-90-R)}

A commonly used self-report instrument for measuring symptoms is the Symptom Check List-90 items-Revised version, SCL-90-R (Derogatis, 1983). The SCL-90-R consists of nine symptom Clusters/subscales that each include 6 - 13 items. The severity of 90 symptoms over the last week is rated on a five-point Likert scale ranging from 0 (not at all) to 4 (extremely). The mean score of all 90 items, the Global Severity Index (GSI), is most often reported in studies that employ the SCL-90-R. The Norwegian version of the SCL-90-R has demonstrated adequate reliability (Bjerke et al., 2014; Vassend, \& Skrondal, 2003).

\subsection{Procedure}

Normed scores were used to calculate IIP-64 structural summary variables. Thus, individual scores were standardized in relation to the mean and standard deviation of the normal reference sample (Monsen et al., 1999). Based on calculations of each patient's Angle (see section on the structural summary above), the clinical sample was divided into Octant Groups. Patient profiles peaking at an Angle of $45^{\circ} \pm 22.5^{\circ}$ (i.e., from $22.5^{\circ}$ to $67.5^{\circ}$ ) were allocated to the Intrusive (NO) Octant Group, those with an Angle of $90^{\circ} \pm 22.5^{\circ}$ were allocated to the Domineering (PA) Octant Group, and so on. Octant Groups constitute the main independent group variable of the following analyses.

The Regional Committee for Medical Research Ethics for Eastern Norway approved the study (Ref. 1.2005.1305).

\subsection{Statistical Analyses}

In addition to carrying out descriptive statistics calculations, ANCOVA analyses were conducted, with Affiliation, Self-Acceptance, and Self-Hostility as dependent variables, interpersonal octant groups as factors, and GSI, IIP Global, and Intensity as model covariates (SPSS 18.0), to test the hypothesized relationships between self-relatedness and interpersonal problems. When significant differences among Octant Groups were found $(p<$ 0.05), post hoc pairwise comparisons with Bonferroni adjustments were conducted to determine how the Octant Groups differed with respect to the Self-Affiliation variable. Effect size estimates were based on partial eta squared and Cohen/s $d$ values. 


\section{Results}

The data were compared with a Norwegian normal reference sample $(N=302)$ with a mean age of 33 years (range 18 - 65) and with female respondents comprising 60\% of the sample (Monsen, Kallerud, Eilertsen, \& von der Lippe, 1999). The clinical sample reported significantly more interpersonal problems and symptoms, compared to the normal reference sample. The mean IIP-64 score (with standard deviations shown in parentheses) for the overall level of interpersonal problems in the clinical sample was $1.42(0.54)$. The comparison with the Norwegian reference sample, $M=0.97$ (0.58), yielded a standardized mean difference (Cohen's $d$; Cohen, 1988) of 0.87 , indicating a significant difference with patients reporting substantially more relationship issues than the general population. The overall level of psychiatric symptoms, as measured by the SCL-90-R (General Severity Index-GSI), was 1.50 (0.72). The comparison with the Norwegian reference sample, $M=0.45$ (0.40), yielded a standardized mean difference (Cohen's $d$ ) of 1.60, signifying high levels of symptom distress in the patient sample.

Table 1 presents the correlations between the self-relatedness variables, interpersonal variables, and symptoms (GSI).

As expected, the correlations were generally high and within a range that justifies the inclusion of the IIP Global, Intensity and GSI as covariates in the analysis of possible associations between the self-relatedness variables and interpersonal Octant Groups.

Table 1 also demonstrates that the correlation between the Self-Accepting and Self-Hostile components yielded a Pearson $r$ of -0.45 , which is much lower than would be expected for components positioned on opposite ends of the Affiliation dimension. This supports the notion that these two variables represent something more than two extremes of the same dimension (cf. our first research aim).

Table 2 presents the SASB Introject Cluster scores, together with the Affiliation, Self-Acceptance, and SelfHostility measures, in the clinical sample and the normal reference sample. The samples differed significantly on all measures. The effect size estimates indicated, as expected, a large variation on the Affiliation dimension $(d=1.28)$. The clinical sample scored significantly lower on Self-Acceptance $(d=-0.97)$ and significantly higher on Self-Hostility $(d=1.19)$ relative to the reference sample. Differences between the samples in Cluster scores representing the Interdependence dimension varied from negligible and small effect sizes for Clusters 1 (Self-Emancipate $[d=-0.17]$ ) and 4 (Self-Protect $[d=-0.28]$ ) to a moderate effect size for Cluster 8 (SelfIgnore $[d=0.72])$.

Table 3 presents overall and unique contributions of segments of the model in terms of the explained variance of Affiliation and its two subcomponents, Self-Acceptance, and Self-Hostility. The overall model explained $41.9 \%$ of the variance in Affiliation, $40.7 \%$ of the variance in Self-Hostility, and $23.4 \%$ of the variance in SelfAcceptance. The IIP Global and GSI accounted for the majority of explained variance in Self-Hostility and overall Affiliation. The Intensity and Octant group contributed significantly to the variance in Affiliation and, to a greater degree, the Self-Accepting sub-component. The variance in Self-Hostility appeared unaffected by the Intensity and Octant Groups. These results indicate that the effect from Octant Group on Affiliation is due to its subcomponent Self-Acceptance.

The fourth hypothesis focused on specific associations between predominant interpersonal problems (Octant Groups) and forms of self-relatedness. Table 4 shows estimated marginal mean scores with respect to SelfAcceptance and Self-Hostility among the Octant Groups. The Affiliation (i.e. difference) scores are omitted in

Table 1. Pearson correlation coefficients between all pairs of variables examined in the study.

\begin{tabular}{cccccc}
\hline & Affiliation & Self-Accept. & Self-Hostility & IIP Global & Intensity \\
\hline Affiliation & - & 0.82 & -0.88 & -0.56 & -0.34 \\
Self-Accept. & - & - & -0.45 & -0.37 & -0.30 \\
Self-Hostility & - & - & - & -0.57 & 0.28 \\
IIP Global & - & - & - & -0.36 & -0.51 \\
Intensity & - & - & - & - & - \\
GSI & - & - & - & -
\end{tabular}

Note: All correlations are significant at the 0.01 level (two-tailed). 
Table 2. SASB Introject: Characteristics of the clinical sample compared to the normal reference sample.

\begin{tabular}{|c|c|c|c|c|c|c|c|}
\hline & & \multicolumn{2}{|c|}{ Clin. sample $(n=958)$} & \multicolumn{2}{|c|}{ Ref. sample $(n=301)$} & \multirow{2}{*}{$F$} & \multirow{2}{*}{ Cohen's $d$} \\
\hline & & $M$ & $S D$ & $M$ & $S D$ & & \\
\hline Self-Emancip. & Cluster 1 & 3.61 & 1.77 & 3.90 & 1.32 & $6.81^{* *}$ & -0.17 \\
\hline Self-Affirm & Cluster 2 & 3.60 & 2.30 & 6.34 & 1.90 & $352.24^{* * *}$ & -1.24 \\
\hline Self-Love & Cluster 3 & 4.25 & 2.13 & 5.99 & 1.86 & $161.80^{* * *}$ & -0.84 \\
\hline Self-Protect & Cluster 4 & 5.66 & 1.77 & 6.15 & 1.65 & $18.41^{* * *}$ & -0.28 \\
\hline Self-Control & Cluster 5 & 4.80 & 2.24 & 3.76 & 1.94 & $51.82^{* * *}$ & 0.48 \\
\hline Self-Blame & Cluster 6 & 4.59 & 2.69 & 1.76 & 1.92 & $287.19^{* * *}$ & 1.12 \\
\hline Self-Attack & Cluster 7 & 4.52 & 2.29 & 1.97 & 1.58 & $324.79^{* * * *}$ & 1.19 \\
\hline Self-Ignore & Cluster 8 & 3.43 & 2.39 & 1.83 & 1.55 & $119.72^{* * *}$ & 0.72 \\
\hline Self-Accept. & - & 74.79 & 30.18 & 102.92 & 25.14 & $214.76^{* * *}$ & -0.97 \\
\hline Self-Hostility & - & 71.36 & 36.09 & 31.48 & 24.02 & $322.50^{* * * *}$ & 1.19 \\
\hline Affiliation & - & $3.43^{\mathrm{a}}$ & 56.57 & 71.44 & 40.80 & $373.92^{* * *}$ & -1.28 \\
\hline
\end{tabular}

Note. Cohen's $d$ with pooled standard deviations: $\left(M_{1}-M_{2}\right) / \sqrt{\left[\left(S D_{1}^{2} *\left(n_{1}-1\right)+S D_{2}^{2} *\left(n_{2}-1\right)\right) /\left(n_{1}+n_{2}-2\right)\right]}$; ${ }^{\text {Range: }} 300.15$ (Min: -156.75 , Max: 143.40). $\stackrel{* *}{p}<0.01, \stackrel{* * *}{p} p<0.001$.

Table 3. Contributions of IIP-64 structural summary variables and GSI to the explained variance in SASB affiliation (AFF) and its two components.

\begin{tabular}{|c|c|c|c|c|c|c|}
\hline & & \multicolumn{5}{|c|}{ Variance } \\
\hline & & \multirow{2}{*}{ Total } & \multicolumn{3}{|c|}{ Unique } & \multirow[b]{2}{*}{ GSI } \\
\hline & & & IIP Global & Intensity & Octant Gr & \\
\hline \multirow[t]{2}{*}{ AFF } & $F(d f)$ & $68.27(10)^{* * * *}$ & $73.87(1)^{* * *}$ & $18.58(1)^{* * * *}$ & $4.08(7)^{*}$ & $\overline{-}^{-} 115.45(1)^{* * *}$ \\
\hline & $\%$ & 41.9 & 7.2 & 1.9 & 2.9 & 10.9 \\
\hline \multirow[t]{2}{*}{ Self-Acc } & $F(d f)$ & $29.00(10)^{* * * *}$ & $14.92(1)^{* * *}$ & $26.82(1)^{* *}$ & $7.13(7)^{* * *}$ & $34.44(1)^{* * *}$ \\
\hline & $\%$ & 23.4 & 1.6 & 2.8 & 5.0 & 3.5 \\
\hline \multirow[t]{2}{*}{ Self-Hos } & $F(d f)$ & $65.08(10)^{* * *}$ & $93.47(1)^{* * * *}$ & $3.13(1)$ & $0.67(7)$ & $123.19(1)^{* * * *}$ \\
\hline & $\%$ & 40.7 & 9.0 & 0.3 & 0.5 & 11.5 \\
\hline
\end{tabular}

Note: The results are based on three separate ANCOVA analyses: AFF/Self-Acceptance/Self-Hostility = IIP Global + Intensity + Octant Group + GSI. AFF = Affiliation; Self-Acc = Self-Acceptance; Self-Hos = Self-Hostility. Intensity=IIP Intensity; Oct Gr = Octant Group. GSI = General Severity Index of SCL-90-R. F-values, degrees of freedom $(d f)$, statistical significance and percent explained variance (partial eta squared: $\left.\eta^{2} \times 100\right)$ are displayed. ${ }^{*} p<0.05,{ }^{* *} p<0.01,{ }^{* * *} p<0.001$.

Table 4 (cf. the results above). $F$-values, $p$-values, partial eta squared values, and pairwise comparisons (with Bonferroni adjustments) are presented.

It appeared that the Octant Group effect was due to differences in the Self-Accepting component. Patients who were categorized under the right-hand and upper right-hand Octant Groups (LM and NO) scored significantly higher on the Self-Acceptance measure than patients of the three Octant Groups categorized under the lower left-hand area of the IPC. Additionally, patients of the Vindictive (BC) Octant Group appeared significantly more self-accepting than those of the Socially Inhibited (FG) group. We found overall high scores on Self-Hostility, but no significant differences between Octant Groups. Variations in Self-Acceptance across IIP Octant Groups are presented graphically in Figure 3. 
Table 4. SASB Introject scores among IIP octant groups. Estimated marginal means from ANCOVAs, with IIP global, intensity and GSI as covariates.

\begin{tabular}{ccccc}
\hline & $n(958)$ & $\%$ & Self-Accept. & Self-Hostility \\
\hline Domineering PA & 62 & 6.5 & 78.14 & 70.69 \\
Vindictive BC & 99 & 10.3 & 79.39 & 68.62 \\
Cold DE & 106 & 11.1 & 69.86 & 71.34 \\
Socially Inhib FG & 146 & 15.2 & 66.86 & 71.60 \\
Nonassertive HI & 229 & 23.9 & 71.15 & 72.98 \\
Overly Accom JK & 171 & 17.9 & 76.11 & 73.51 \\
Self-Sacrificing LM & 93 & 9.7 & 86.53 & 67.50 \\
Intrusive NO & 52 & 5.4 & 84.96 & 69.45 \\
$F(7,947)$ & - & - & 7.13 & 0.67 \\
$p$ & - & - & $<0.001$ & 0.70 \\
Partial eta squared & - & - & 0.050 & 0.005 \\
Pairwise Bonferroni & - & LM, NO > DE, FG, HI & NS
\end{tabular}

Note. $\mathrm{PA}=$ Domineering, $\mathrm{BC}=$ Vindictive, $\mathrm{DE}=$ Cold, $\mathrm{FG}=$ Socially Inhibited, $\mathrm{HI}=$ Nonassertive, $\mathrm{JK}=$ Overly Accommodating, $\mathrm{LM}=$ Self-Sacrificing, $\mathrm{NO}=$ Intrusive.

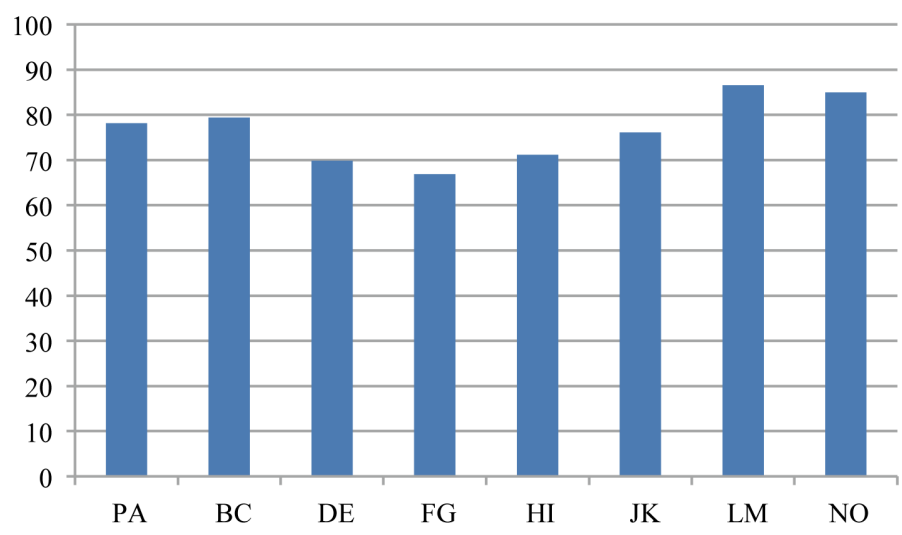

Figure 3. Self-Acceptance scores among interpersonal Octant Groups. $\mathrm{PA}=$ Domineering, $\mathrm{BC}=$ Vindictive $, \mathrm{DE}=\mathrm{Cold}, \mathrm{FG}=$ Socially Inhibited, $\mathrm{HI}=$ Nonassertive, $\mathrm{JK}=$ Overly Accommodating, $\mathrm{LM}=$ SelfSacrificing, $\mathrm{NO}=$ Intrusive.

\section{Discussion}

This study tested hypothesized associations between self-reported forms of self-relatedness and interpersonal problems among psychiatric outpatients. We expected to find 1) support for the notion that splitting the SASB Introject Affiliation variable into its Self-Hostile and Self-Accepting components may be important in assessment of self-relatedness; 2) that the clinical sample would exhibit Affiliation scores indicating significantly poorer self-image than the normal reference sample; 3) that all the IIP-variables (IIP Global, Intensity, and Octant Group), in addition to general symptom distress, would contribute significantly to the variance of the SASB Introject variables (Affiliation, Self-Hostility, and Self-Acceptance); and 4) that patients with interpersonal problems of the domineering kind would report a less poor self-image than those with interpersonal problems of the more submissive type.

Concerning our first hypothesis: Table 3 and Table 4 show that the two components of Affiliation associate 
differentially with the IIP variables. The implications of this finding are discussed later in this section.

The clinical outpatient sample presented considerably more negative forms of self-relatedness than the normal reference sample. Clusters 2 and 3 (on the warm side) and Clusters 6 and 7 (on the cold side) displayed the greatest effect sizes, indicating that the largest deviations from the "normal" self-image can be found along the Affiliation dimension. This supports our second hypothesis and confirms the assumption that the Affiliation dimension is the most pertinent to clinical assessments of the patients' self-relatedness.

Concerning the hypothesized contributions to the variance of the self-relatedness variables, we found that two of the interpersonal variables (Octant Group and Intensity) did not contribute to the variance of Self-Hostility. Thus, our hypothesis that all the interpersonal variables would contribute to the variance in Affiliation was supported, but this was not the case regarding its subcomponents. It seems that the unique effect from Octant Group, more specifically, is due to the effect on the Self-Accepting component of Affiliation.

Accordingly, the hypothesized association between the high Agency dimension of the IIP (i.e., domineering types of interpersonal problems) and a more accepting self-relatedness applied to the Self-Accepting but not for the Self-Hostile component. Patients allocated to the two high Agency Octant Groups, Intrusive (NO) and Vindictive (BC), presented significantly higher Self-Acceptance scores than patients in the Socially Inhibited (FG) Octant Group. On the other hand, we also found that a warm interpersonal style, Self-Sacrificing (LM), was associated with an accepting relationship with oneself, whereas a cold interpersonal style was associated with low Self-Acceptance. These findings may suggest different forms of positive Self-Acceptance: one reflecting parallelism between relations with oneself and relations with others ("I'm okay, you're okay") and another whereby more defensively motivated positive Self-Acceptance combines with a Domineering-Vindictive relation toward others. This is clinically interesting: a relatively Self-Accepting form of self-relatedness may be associated with relatively benign interpersonal problems on the warmer side of the IPC but might also be defensively motivated and associated with more severe interpersonal problems attributed to the cold/domineering side of the IPC.

Awareness of the two components of Affiliation, as demonstrated in the present article, can have prognostic and therapeutic relevance. Previous studies have suggested that different self-image components exhibit dissimilar patterns of change as a result of psychotherapy (Granberg \& Armelius, 2003; Halvorsen \& Monsen, 2006; Halvorsen \& Monsen, 2007; Junkert-Tress, Schnierda, Hartkamp, Schmitz, \& Tress, 2001; Malmgren-Olsson, Armelius, \& Armelius, 2001). Halvorsen and Monsen (2006) claimed that a change in Self-Hostility tendencies over long-term treatment is easier to achieve and occurs earlier in the treatment process than changes toward increased Self-Acceptance. Discarding Self-Hostile attitudes may promote optimism and pave the way for more fundamental changes in the realm of Self-Acceptance, which entails a more complex process that often requires a greater amount of time. These differences may be attributable to different underlying features of the two subcomponents of Affiliation. The Self-Hostile component is likely to be associated with negative affect (e.g., affect associated with anxiety and depression), which often improves rapidly with or without therapy. One might say that the Self-Hostile component displays more state-like features. Self-Acceptance, on the other hand, may be associated with more stable, trait-like beliefs, which likely require more extensive efforts to change. This assumption is also supported by the findings presented in Table 3, which showed that the two global indicators of distress (IIP global and SCL/GSI) accounted for more than $20 \%$ of the variance in Self-Hostility, but only approximately $5 \%$ of the variance in Self-Acceptance. This is in line with the finding that Self-Acceptance seems to associate with specific interpersonal types (i.e. personalities), which are likely to change more slowly during treatment.

Pre-treatment assessments based on interpersonal problems, self-relatedness, and symptom self-reports may help guide effective diagnosis, prognosis and treatment planning strategies. Patients of the Socially Inhibited and Nonassertive Octant Groups (comprising nearly 40\% of the present sample) share common features, that is, high levels of interpersonal problems in general (IIP Global), high Intensity of interpersonal problems, high levels of phobic anxiety, and low levels of hostility and paranoid ideation (Bjerke et al., 2011, 2014). The present study shows that these patients also are the most affected with regard to poor Self-Acceptance, which probably indicates that these patients will need more time to change than patients located on the warm side of the IPC.

\section{Conclusion}

This study concerns associations between self-relatedness and interpersonal problems, but does not address the interesting questions of "what comes first", how the interrelationship between relationship to self and to others 
influence each other, either in vicious circles or more benign, therapeutic manners. And: where to start in therapy? On group basis, this needs further research. For the individual patient and therapist, this might be a focus for their common striving to make the patient better.

We note a number of other study limitations. Large subgroups of outpatients were initially excluded, including emergency cases, non-native Norwegian-speaking individuals, and previously registered patients. It is possible that our findings would have differed if acute and/or inpatient psychiatric individuals had been included in the study. The structural summary method implies that when placing a certain individual interpersonal profile in one specific Octant Group, high scores in adjacent (or more distant) areas of "circular space" may be obscured. It is also possible that a patient with high subscale scores in, for instance, the Intrusive and Vindictive IPC domains but low Domineering scores would fall under the Domineering Octant Group as a result of structural summary calculations. This would have generated a less prototypic interpersonal problems profile and a reduced goodness of fit index (GOF-cf. p. 7). However, GOF values were generally acceptable for the profiles included in the present analyses (Bjerke et al., 2011). Furthermore, as we focused on the Affiliation dimension of the SASB Introject, we omitted the Interdependence dimension from our analyses. A more comprehensive utilization of the SASB Introject and study of its associations with the IIP may be pursued in further research.

\section{Implications}

In sum, there are important implications of our findings for practitioners conducting psychological assessment. It appears to be important when assessing self-image with the SASB Introject surface to be aware of the fact that Self-Hostility and Self-Acceptance are less strongly associated than one would theoretically expect. In addition, these two components appear to be differently related to interpersonal problems. Our findings indicate that nuanced assessment of interpersonal problems may give rise to quite specific hypotheses about the person's self-relatedness.

\section{References}

Alden, L. E., Wiggins, J. S., \& Pincus, A. L. (1990). Construction of Circumplex Scales for the Inventory of Interpersonal Problems. Journal of Personality Assessment, 55, 521-536. http://dx.doi.org/10.1207/s15327752jpa5503\&4_10

Benjamin, L. S. (1974). Structural Analysis of Social Behavior. Psychological Review, 81, 392-425. http://dx.doi.org/10.1037/h0037024

Benjamin, L. S. (1996). A Clinician-Friendly Version of the Interpersonal Circumplex: Structural Analysis of Social Behavior (SASB). Journal of Personality Assessment, 66, 248-266. http://dx.doi.org/10.1207/s15327752jpa6602_5

Bjerke, E., Hansen, R. S., Solbakken, O. A., \& Monsen, J. T. (2011). Interpersonal Problems among 988 Norwegian Psychiatric Outpatients. A Study of Pre-Treatment Self-Reports. Comprehensive Psychiatry, 52, 273-279. http://dx.doi.org/10.1016/j.comppsych.2010.07.004

Bjerke, E., Solbakken, O. A., \& Monsen, J. T. (2014). Are There Specific Relationships between Symptom Patterns and Interpersonal Problems among Psychiatric Outpatients? Journal of Personality Assessment, 96, 237-244. http://dx.doi.org/10.1080/00223891.2013.834441

Cohen, J. (1988). Statistical Power Analysis for the Behavioral Sciences. Hillsdale, NJ: Lawrence Erlbaum Association.

Derogatis, L. R. (1983). SCL-90-R: Administration, Scoring and Procedures: Manual II. Baltimore, MD: Clinical Psychometric Research.

Granberg, Å., \& Armelius, K. (2003). Change of Self-Image in Patients with Neurotic, Borderline and Psychotic Disturbances. Clinical Psychology \& Psychotherapy, 10, 228-237. http://dx.doi.org/10.1002/cpp.371

Gurtman, M. B. (2009). Exploring Personality with the Interpersonal Circumplex. Social and Personality Psychology Compass, 3, 601-619. http://dx.doi.org/10.1111/j.1751-9004.2009.00172.x

Gurtman, M. B., \& Balakrishnan, J. D. (1998). Circular Measurement Redux: The Analysis and Interpretation of Interpersonal Circle Profiles. Clinical Psychology: Science and Practice, 5, 344-360. http://dx.doi.org/10.1111/j.1468-2850.1998.tb00154.x

Gurtman, M. B., \& Pincus, A. L. (2003). The Circumplex Model: Methods and Research Applications. In J. A. Schinka, \& W. F. Velicer (Eds.), Comprehensive Handbook of Psychology (pp. 407-428). New York: Wiley.

Guttman, L. (1954). A New Approach to Factor Analysis: The Radex. In P. F. Lazarsfeld (Ed.), Mathematical Thinking in the Social Sciences (pp. 258-348). New York: Free Press.

Halvorsen, M. S., \& Monsen, J. T. (2006). The Influence of Pre-Treatment Patient Characteristics and Treatment Duration on Change in Self-Image. In M. S. Halvorsen (Ed.), Self-Image and Psychotherapy: Measurement, Predictor and Outcome 
Issues (No. 54, Paper IV). (Doctoral Dissertation). Oslo: University of Oslo.

Halvorsen, M. S., \& Monsen, J. T. (2007). Self-Image as a Moderator of Change in Psychotherapy. Psychotherapy Research, 17, 205-217. http://dx.doi.org/10.1080/10503300600608363

Henry, W. P. (1996). Structural Analysis of Social Behavior as a Common Metric for Programmatic Psychopathology and Psychotherapy Research. Journal of Consulting and Clinical Psychology, 64, 1263-1275. http://dx.doi.org/10.1037/0022-006X.64.6.1263

Henry, W. P., Schacht, T. E., \& Strupp, H. H. (1986). Structural Analysis of Social Behavior: Application to a Study of Interpersonal Process in Differential Psychotherapeutic Outcome. Journal of Consulting and Clinical Psychology, 54, 27-31. http://dx.doi.org/10.1037/0022-006X.54.1.27

Henry, W. P., Schacht, T. E., \& Strupp, H. H. (1990). Patient and Therapist Introject, Interpersonal Process, and Differential Psychotherapy Outcome. Journal of Consulting and Clinical Psychology, 58, 768-777. http://dx.doi.org/10.1037/0022-006X.58.6.768

Hilliard, R. B., Henry, W. P., \& Strupp, H. H. (2000). An Interpersonal Model of Psychotherapy: Linking Patient and Therapist Developmental History, Therapeutic Process, and Types of Outcome. Journal of Consulting and Clinical Psychology, 68, 125-133. http://dx.doi.org/10.1037/0022-006X.68.1.125

Horowitz, L. M. (2004). Interpersonal Foundations of Psychopathology. Washington DC: American Psychological Association Interpersonal. http://dx.doi.org/10.1037/10727-000

Horowitz, L. M., Alden, L., Wiggins, J., \& Pincus, A. (2000). Inventory of Interpersonal Problems Manual. San Antonio, TX: The Psychological Corporation.

Horowitz, L. M., Rosenberg, S. E., Baer, B. A., Ureno, G., \& Villasenor, V. S. (1988). Inventory of Interpersonal Problems: Psychometric Properties and Clinical Applications. Journal of Consulting and Clinical Psychology, 56, 885-892. http://dx.doi.org/10.1037/0022-006X.56.6.885

Horowitz, L. M., Wilson, K. R., Turan, B., Zolotsev, P., Constantino, M. J., \& Henderson, L. (2006). How Interpersonal Motives Clarify the Meaning of Interpersonal Behavior: A Revised Circumplex Model. Personality and Social Psychology Review, 10, 67-86. http://dx.doi.org/10.1207/s15327957pspr1001_4

Junkert-Tress, B., Schnierda, U., Hartkamp, N., Schmitz, N., \& Tress, W. (2001). Effects of Short-Term Dynamic Psychotherapy for Neurotic, Somatoform, and Personality Disorders: A Prospective 1-Year Follow-Up Study. Psychotherapy Research, 11, 187-200.

Leary, T. (1957). Interpersonal Diagnosis of Personality. New York: Ronald Press Co.

Mahler, M., Pine, F., \& Bergman, A. (1975). The Psychological Birth of the Infant. New York: Basic Books.

Malmgren-Olsson, E. B., Armelius, B. A., \& Armelius, K. (2001). A Comparative Outcome Study of Body Awareness Therapy, Feldenkrais, and Conventional Physiotherapy for Patients with Nonspecific Musculoskeletal Disorders: Changes in Psychological Symptoms, Pain, and Self-Image. Physiotherapy Theory and Practice, 17, 77-95. http://dx.doi.org/10.1080/095939801750334167

Monsen, J. T., Hagtvet, K. A., Havik, O. E., \& Eilertsen, D. E. (2006). Circumplex Structure and Personality Disorder Correlates of the Interpersonal Problems Model (IIP-C): Construct Validity and Clinical Implications. Psychological Assessment, 18, 165-173. http://dx.doi.org/10.1037/1040-3590.18.2.165

Monsen, J. T., Kallerud, J. E., Eilertsen, D. E., \& von der Lippe, A. (1999). The Data Base for the Norwegian Multisite Study of Process and Outcome in Psychotherapy, User's Manual. Oslo: University of Oslo.

Monsen, J. T., von der Lippe, A. L., Havik, O. E., Halvorsen, M. S., \& Eilertsen, D. E. (2007). Validation of the SASB Introject Surface in a Norwegian Clinical and Nonclinical Sample. Journal of Personality Assessment, 88, $235-245$. http://dx.doi.org/10.1080/00223890701268108

Pincus, A. L., \& Gurtman, M. B. (2006). Interpersonal Theory and the Interpersonal Circumplex. Evolving Perspectives on Normal and Abnormal Personality. In S. Strack (Ed.), Differentiating Normal and Abnormal Personality (2nd ed., pp. 83111). New York: Springer.

Pincus, A. L., Gurtman, M. B., \& Ruiz, M. A. (1998). Structural Analysis of Social Behavior (SASB): Circumplex Analyses and Structural Relations with the Interpersonal Circle and the Five-Factor Model of Personality. Journal of Personality and Social Psychology, 74, 1629-1645. http://dx.doi.org/10.1037/0022-3514.74.6.1629

Pincus, A. L., Newes, S. L., Dickinson, K. A., \& Ruiz, M. A. (1998). A Comparison of Three Indexes to Assess the Dimensions of Structural Analysis of Social Behavior. Journal of Personality Assessment, 70, 145-170. http://dx.doi.org/10.1207/s15327752jpa7001_10

Ruiz, M. A., Pincus, A. L., Borkovec, T. D., Echemendia, R. J., Castonguay, L. G., \& Ragusea, S. A. (2004). Validity of the Inventory of Interpersonal Problems for Predicting Treatment Outcome: An Investigation with the Pennsylvania Practice Research Network. Journal of Personality Assessment, 83, 213-222. http://dx.doi.org/10.1207/s15327752jpa8303_05 
Sullivan, H. S. (1953). The Interpersonal Theory of Psychiatry. New York: Norton.

Svartberg, M., Seltzer, M. H., \& Stiles, T. (1996). Self-Concept Improvement during and after Short-Term Anxiety-Provoking Psychotherapy: A Preliminary Growth Curve Study. Psychotherapy Research, 6, 43-55. http://dx.doi.org/10.1080/10503309612331331568

Vassend, O., \& Skrondal, A. (2003). Interpretation of the SCL-90-R. A Psychometric Study Based on a Norwegian National Sample. Oslo: Royal Norwegian Air Force, Institute of Aviation Medicine.

Wiggins, J. S. (1979). A Psychological Taxonomy of Trait Descriptive Terms: The Interpersonal Domain. Journal of Personality and Social Psychology, 37, 395-412. http://dx.doi.org/10.1037/0022-3514.37.3.395

Wiggins, J. S. (1996). An Informal History of the Interpersonal Circumplex Tradition. Journal of Personality Assessment, 66, 217-233. http://dx.doi.org/10.1207/s15327752jpa6602 2

Wright, A. G. C., Pincus, A. L., Conroy, D. E., \& Hilsenroth, M. J. (2009). Integrating Methods to Optimize Circumplex Description and Comparison of Groups. Journal of Personality Assessment, 91, 311-322. http://dx.doi.org/10.1080/00223890902935696

\section{Submit or recommend next manuscript to SCIRP and we will provide best service for you:}

Accepting pre-submission inquiries through Email, Facebook, Linkedin, Twitter, etc A wide selection of journals (inclusive of 9 subjects, more than 200 journals)

Providing a 24-hour high-quality service

User-friendly online submission system

Fair and swift peer-review system

Efficient typesetting and proofreading procedure

Display of the result of downloads and visits, as well as the number of cited articles

Maximum dissemination of your research work

Submit your manuscript at: http://papersubmission.scirp.org/ 